\title{
Mycoplasma pneumoniae and Chlamydia pneumoniae infections in children with pneumonia
}

\author{
S. Esposito*, F. Blasi\#, F. Bellini", L. Allegra\#, N. Principi*, and the Mowgli Study Group
}

Mycoplasma pneumoniae and Chlamydia pneumoniae infections in children with pneumonia. S. Esposito, F. Blasi, F. Bellini, L. Allegra, N. Principi, and the Mowgli Study Group. C ERS Journals Ltd 2001.

ABSTRACT: The most common clinical signs, host responses and radiographic patterns were studied in 203 Italian children hospitalized for community-acquired pneumonia in order to clarify the role of clinical and radiological characteristics in the diagnosis of Mycoplasma pneumoniae and/or Chlamydia pneumoniae infections.

Antibody measurements in paired sera and polymerase chain reaction on nasopharyngeal aspirates were used to establish the diagnoses of acute $M$. pneumoniae and $C$. pneumoniae infection, and the aetiologic data were correlated with the clinical, laboratory and radiographic data obtained on admission.

No significant association was observed between evidence of $M$. pneumoniae and/or $C$. pneumoniae infection and periods of episode during the year, mean age of the study subjects, individual symptoms, physical findings or laboratory test results. Furthermore, no significant correlation was observed in relation to the radiological findings and $M$. pneumoniae and/or $C$. pneumoniae infection.

This study shows that neither clinical findings nor laboratory parameters distinguished Mycoplasma pneumoniae and/or Chlamydia pneumoniae infection in children with pneumonia. Radiological findings also have a limited capacity to differentiate aetiologic agents. The priorities for future research include the development of rapid, easily accessible and cost-effective diagnostic tests useful for each episode of pneumonia in children.

Eur Respir J 2001; 17: 241-245.

\begin{abstract}
*Paediatric Dept I, "Institute of Respiratory Diseases, Istituto di Ricerca e di Cura a Carattere Scientifico, Maggiore Hospital and "Institute of Radiology, San Paolo Hospital, University of Milan, Milan, Italy
\end{abstract}

Correspondence: N Principi, Paediatric Department I, University of Milan, via Commenda, 9, 20122, Milan, Italy. Fax: 39-02-55195341

\section{Keywords: Children}

Chlamydia pneumoniae

Mycoplasma pneumoniae pneumonia

Received: March 202000

Accepted after revision July 252000

This work was supported in part by Abbott SpA, Italy.
Recent studies reported that Mycoplasma pneumoniae and Chlamydia pneumoniae play a significant role as causes of community-acquired pneumonia in children of all ages [1-3]. Since these atypical pathogens may cause chronic colonisation of the respiratory tract $[4,5]$ and are not susceptible to the $\beta$-lactam regularly used for the treatment of paediatric pneumonia [6,7], rapid diagnostic tests are important. The designation of specific clinical and radiological features to an aetiologic agent has been common practice $[8,9]$, but recent data have cast doubt on the specificity of these observations when comparing individual clinical manifestations [10-13]. The diagnosis of $M$. pneumoniae and $C$. pneumoniae infections relies on serology, cultures and polymerase chain reactions (PCR), all of which are clinically impractical $[14,15]$; it would therefore be beneficial if clinical characteristics, nonspecific inflammatory parameters and the type of infiltration in a chest radiograph could be used to identify these pathogens.

There are few data concerning the possibility of differentiating $M$. pneumoniae and $C$. pneumoniae infections on the basis of presenting manifestations in children with pneumonia. Therefore, the most common clinical signs, host responses and radiographic patterns in 203 Italian children hospitalized for communityacquired pneumonia were studied, in order to clarify further the clinical, biological and radiological characteristics of acute $M$. pneumoniae and/or $C$. pneumoniae infections. The most advanced set of microbiological methods were used to establish the diagnoses of $M$. pneumoniae and $C$. pneumoniae infection, and correlated the aetiologic data with the clinical, laboratory and radiographic data obtained on admission.

\section{Materials and methods}

\section{Study subjects}

The study involved 203 children aged $2-14$ yrs, who were admitted to hospitals in 21 Italian municipalities between May 1998 and April 1999. The patients were hospitalized on the basis of the clinical decisions of the physicians on duty. Previously healthy male and female children aged 2-14 yrs, with signs, symptoms and chest radiographs consistent with community-acquired pneumonia, were considered eligible for inclusion. Exclusion criteria included severe concomitant diseases (neoplasia, kidney or liver disease, immunodepression, cardiovascular disease, malabsorption syndrome), nosocomial acquired infections and use of antibiotics in the $48 \mathrm{~h}$ before enrolment. The study protocol was approved by the Institutional Review Boards at each of 
the centres and informed consent was obtained from the parents or legal guardians of each child before enrolment.

\section{Methods}

Upon admission, systematic recordings were made of the patients' medical history, rectal temperature, respiratory frequency and auscultation findings. This information was collected on a detailed data form, prepared in order to standardize the interpretation of clinical findings. Blood samples were taken for host response measurements: white blood cell (WBC) count, serum C-reactive protein (CRP), and erythrocyte sedimentation rate (ESR). Total and differential WBC counts were determined using an automatic cell counter, serum CRP concentration was measured by means of immunonephelometric method and ESR using the Westergren method. Serum was collected for $M$. pneumoniae and $C$. pneumoniae antibody measurements: immunoglobulin (Ig)M and $\operatorname{IgG}$ to $M$. pneumoniae were tested by means of an enzyme-linked immunosorbent assay (Pantec, Turin, Italy), whereas $\operatorname{IgM}, \operatorname{IgG}$ and $\operatorname{IgA}$ to $C$. pneumoniae were evaluated by means of microimmunofluorescence (Labsystems, Helsinki, Finland). Nasopharyngeal aspirates were also obtained, immediately immersed in sucrose-phosphateglutamate transport medium, and stored at $-70^{\circ} \mathrm{C}$ until assayed for the presence of $M$. pneumoniae and $C$. pneumoniae deoxyribonucleic acid (DNA). PCR for both pathogens was performed as previously described $[16,17]$. Chest radiographs (erect posteroanterior and lateral view) were taken in the hospital of admission and were then centrally reviewed by an experienced radiologist who was naive to the patients' clinical history or laboratory data. Seven radiological features (hyperinflation, peribronchial wall thickening, perihilar linear opacities, reticulo-nodular infiltrate, segmental or lobar consolidation, bilateral consolidations and pleural effusion) were recorded as present or absent [18]. Radiographic interpretation alternatives were not mutually exclusive and in each radiogram, more than one category could be checked. Between four and six weeks after admission, repeat blood samples for $M$. pneumoniae and $C$. pneumoniae antibody measurements were taken during the convalescent evaluation.

Acute $M$. pneumoniae and/or C. pneumoniae infection was diagnosed if the patient had a significant antibody response to one of the pathogens in paired sera (IgM antibody, a 4-fold increase in IgG antibody titre, a static IgG antibody titre four times or more than the cut-off of the assay) or if the PCR on nasopharyngeal aspirates was positive for one of them [6].

\section{Analysis}

The data were analysed using SAS Windows v.12 (Cariy, NC, USA). All of the patients were included in the analysis. For all of the statistical tests, a p-value of $<0.05$ was considered statistically significant. Parametric data were compared using analysis of variance (ANOVA) with terms for treatment and tests for multiple comparisons. When the data were not normally distributed, or were non parametric data, the Kruskal-Wallace test was used. Categorical data were analysed using contingency table analysis and the Chi-squared or Fisher's test.

\section{Results}

Of the 203 enrolled children, $110(54.2 \%)$ were males and $93(45.8 \%)$ were females. The mean \pm SD age of the study population was $5.44 \pm 3.06$ yrs: $96(47.3 \%)$ children were aged $2-4$ years, $58(28.6 \%) 5-7$ years, and $49(24.1 \%) 8-14$ years old. Sixty-eight children (33.5\%) had evidence of acute $M$. pneumoniae infection confirmed by serology (18 patients), PCR (4: among them, two were aged 2-4 years and two aged $5-7$ years), or both (46). There were eight $(4 \%)$ children with acute $C$. pneumoniae infection: two identified by serology, three by PCR (among them, two were aged $2-4$ years and one aged 5-7 years) and three by both. A further 11 patients had acute $M$. pneumoniae and $C$. pneumoniae coinfection, confirmed by serology (all with evidence of acute infection for both pathogens) and PCR (ten nasopharyngeal aspirates positive for $M$. pneumoniae DNA and five positive for $C$. pneumoniae). Table 1 shows the distribution of infections by months of the year. Even if minor variations were detected, no significant difference was observed for the different aetiological agents in various months of the year.

The clinical characteristics of the study population at enrolment are summarized in table 2. Age distribution was similar between the groups. Moreover, no significant association was observed between evidence of $M$. pneumoniae and/or $C$. pneumoniae infection and individual symptoms or physical findings. The clinical presentation (including disease onset, the presence of a similar illness in the family, cough, tachypnea, fever, rales and wheezes) was similar in the children with acute $M$. pneumoniae or C. pneumoniae infection and those with acute mixed $M$. pneumoniae and $C$. pneumoniae infection. Fever was the prevalent sign in all aetiological categories. The most common findings on physical examination were rales. The duration of illness and hospitalization was similar in the different groups of children: no significant differences were observed between subjects with acute $M$. pneumoniae or $C$. pneumoniae infection and those with acute mixed $M$. pneumoniae and $C$. pneumoniae infection.

Table 3 shows the laboratory data by aetiological groups. No significant difference was detected in total and differential WBC count, CRP or ESR in the children with acute $M$. pneumoniae or $C$. pneumoniae infection and those with acute mixed $M$. pneumoniae and C. pneumoniae infection. The distribution of WBC, CRP and ESR values was wide within each group.

The radiographic characteristics of the study population are shown in table 4 . No significant correlation was observed in relation to the radiological findings considered as single or associated variables and M. pneumoniae and/or C. pneumoniae infection. 
Table 1.-Distribution of infections by months of the year

\begin{tabular}{lccc}
\hline Months & $\begin{array}{c}\text { Mycoplasma pneumoniae } \\
\text { infection, } \mathrm{n}=68\end{array}$ & $\begin{array}{c}\text { Chlamydia pneumoniae } \\
\text { infection, } \mathrm{n}=8\end{array}$ & $\begin{array}{c}\text { Mixed Mycoplasma pneumoniae } \\
\text { and Chlamydia pneumoniae } \\
\text { infection, } \mathrm{n}=11\end{array}$ \\
\hline May-July $(\mathrm{n}=40)$ & $16(40.0)$ & $1(2.5)$ & $2(5.0)$ \\
August-October $(\mathrm{n}=36)$ & $10(27.7)$ & $2(5.5)$ & $1(2.7)$ \\
November-January $(\mathrm{n}=69)$ & $22(31.8)$ & $1(1.4)$ & $5(7.2)$ \\
February-April $(\mathrm{n}=58)$ & $20(34.4)$ & $4(6.8)$ & $3(5.1)$ \\
\hline
\end{tabular}

Data are presented as number (percentage). No significant differences were observed.

\section{Discussion}

Distinguishing acute $M$. pneumoniae and C. pneumoniae infections may have some merit because they do not respond to $\beta$-lactam antibiotics $[6,7]$ and require specific antimicrobial therapy in order to avoid chronic infections with late sequelae $[4,5]$. These pathogens have been commonly called atypical $[19,20]$ because, in some cases, the clinical presentations were different from those associated with pneumococcal infection [20]. However, most of the information pertaining to the clinical and radiological presentation of atypical bacterial pneumonia comes from adult case series and reports.

This is a large, prospective, multicentre study of paediatric pneumonia performed all around Italy during a one-year study period. Considering that the incidence of infections remained almost stable during different months of the year, the findings do not seem to be related to a clear local outbreak caused by $M$. pneumoniae or $C$. pneumoniae.

The present results are in line with previous data casting doubt on the sensitivity and specificity of clinical and laboratory features in predicting the aetiology of community-acquired pneumonia in children [21-23]. None of the clinical characteristics or laboratory parameters considered by us seem to be unique to atypical bacterial infections, which suggests that they are not useful for therapeutic decision making. It was not possible to predict these aetiological agents only on the basis of the presenting manifestations. WBC, CRP and ESR (all commonly used to measure the severity of the acute phase response) are nonspecific parameters that may be affected by a number of physical, chemical or microbial stimuli [24, 25]. Moreover, in mucosa-limited infections (typical of most community-acquired pneumonia episodes in developed countries) WBC, CRP and ESR values tend to remain low [26]. Their potential for differentiating aetiological agents is therefore highly limited, and considerable overlapping of individual values has been reported in aetiological groups [10, 11].

Radiological diagnosis is subject to the same problems of clinical efficacy as other diagnostic medical tests. The radiographic diagnosis of pneumonia is made on the basis of pulmonary perihilar opacities or infiltrates (airways disease) and/or consolidation (airspace disease) [18, 27]. Like other screening tests, radiological findings are limited in terms of sensitivity, specificity and intra- or inter-observer variability. In the population studied, no feature characterised $M$. pneumoniae and/or $C$. pneumoniae infection and the radiograph cannot be used to predict atypical bacteria infection precisely. These observations are particularly important because, although their appropriateness may be debatable, many clinicians base their decisions regarding the initiation of antibiotics on chest radiographs [28].

Four children positive for M. pneumoniae DNA and three positive for $C$. pneumoniae DNA were considered infected although there was no serologic evidence of acute infection. These children might also be considered simply carriers. However, the number of patients with a significant increase in specific $M$. pneumoniae antibodies was so high that these four children do not affect the conclusions of the study. On the other hand, it is well known that the lack of an immunological response after C. pneumoniae infection may be caused by an immature

Table 2. - Clinical characteristics of the study population at enrollment

\begin{tabular}{lccc}
\hline Characteristics & $\begin{array}{c}\text { Mycoplasma pneumoniae } \\
\text { infection, } \mathrm{n}=68\end{array}$ & $\begin{array}{c}\text { Chlamydia pneumoniae } \\
\text { infection, } \mathrm{n}=8\end{array}$ & $\begin{array}{c}\text { Mixed Mycoplasma pneumoniae and } \\
\text { Chlamydia pneumoniae infection, } \mathrm{n}=11\end{array}$ \\
\hline Mean age, yrs & $6.32 \pm 3.34$ & $5.76 \pm 2.94$ & $6.90 \pm 3.36$ \\
Onset & $41(60.3)$ & $6(75.0)$ & $6(54.5)$ \\
$\quad$ Gradual & $27(39.7)$ & $2(25.0)$ & $5(45.5)$ \\
$\quad$ Acute & $4(5.8)$ & $1(12.5)$ & $1(9.0)$ \\
Similar illness within the family & $44(64.7)$ & $4(50.0)$ & $1(63.6)$ \\
Cough & $8(11.7)$ & $5(62.5)$ & $10(90.9)$ \\
Tachypnea & $58(85.2)$ & $7(87.5)$ & $10(90.9)$ \\
Fever & $60(88.2)$ & $1(12.5)$ & $1(9.0)$ \\
Rales & $10(14.7)$ & $10.75 \pm 4.27$ & $13.00 \pm 5.50$ \\
Wheezes & $13.17 \pm 6.67$ & $6.38 \pm 2.88$ & $6.33 \pm 3.39$ \\
Days of illness & $6.51 \pm 2.74$ & &
\end{tabular}

Data are presented as means \pm SD or number (percentage). No significant differences were observed. 
Table 3. - Laboratory data in the various aetiological groups.

\begin{tabular}{lccc}
\hline Parameter & $\begin{array}{c}\text { Mycoplasma pneumoniae } \\
\text { infection, } \mathrm{n}=68\end{array}$ & $\begin{array}{c}\text { Chlamydia pneumoniae } \\
\text { infection, } \mathrm{n}=8\end{array}$ & $\begin{array}{c}\text { Mixed Mycoplasma pneumoniae and } \\
\text { Chlamydia pneumoniae infection, } \mathrm{n}=11\end{array}$ \\
\hline WBC, cells $\mu \mathrm{LL}^{-1}$ & $13564 \pm 8836$ & $14035 \pm 10173$ & $8074 \pm 2117$ \\
Neutrophils, \% & $66 \pm 17$ & $67 \pm 14$ & $59 \pm 8$ \\
Lymphocytes, \% & $24 \pm 15$ & $23 \pm 13$ & $31 \pm 9$ \\
Monocytes, \% & $7 \pm 5$ & $8 \pm 4$ & $8 \pm 3$ \\
Eosinophils, \% & $2 \pm 2$ & $1 \pm 1$ & $2 \pm 2$ \\
Basophils, \% & $0.4 \pm 0.6$ & $0.7 \pm 0.7$ & $18 \pm 0.3$ \\
$\mathrm{CRP}, \mu \mathrm{g} \cdot \mathrm{dL}-1$ & $53 \pm 83$ & $58 \pm 70$ & $41 \pm 15$ \\
$\mathrm{ESR}, \mathrm{mm} \cdot \mathrm{h}^{-1}$ & $49 \pm 33$ & $49 \pm 25$ & 16 \\
\hline
\end{tabular}

Data are presented as mean values $\pm \mathrm{SD}$; WBC: white blood cell count; CRP: C-reactive protein, ESR: erythrocyte sedimentation rate. No significant differences were observed.

Table 4.-Comparisons of radiographic characteristics of the study population.

\begin{tabular}{lccc}
\hline Finding & $\begin{array}{c}\text { Mycoplasma pneumoniae } \\
\text { infection, \% }(\mathrm{n}=68)\end{array}$ & $\begin{array}{c}\text { Chlamydia pneumoniae } \\
\text { infection, \% }(\mathrm{n}=8)\end{array}$ & $\begin{array}{c}\text { Mixed Mycoplasma pneumoniae } \\
\text { and Chlamydia pneumoniae infection, } \\
\%(\mathrm{n}=11)\end{array}$ \\
\hline Hyperinflation & $10(14.7)$ & $2(25.0)$ & $2(18.2)$ \\
Peribronchial wall thickening & $3(4.4)$ & $2(25.0)$ & $2(18.2)$ \\
Perihilar linear opacities & $41(60.3)$ & $4(50.0)$ & $8(72.7)$ \\
Reticulo-nodular infiltrate & $27(39.7)$ & $1(12.5)$ & $5(45.5)$ \\
Segmental or lobar consolidation & $19(27.9)$ & $3(37.5)$ & 0 \\
Bilateral consolidations & $5(7.4)$ & 0 & 0 \\
Pleural effusion & $4(5.9)$ & 0 & $(18.2)$ \\
\hline
\end{tabular}

No significant differences were observed.

ability to produce a specific humoral response or poor antigenic stimulation in children [29]. Earlier prospective studies in paediatric patients with respiratory infections suggest that over $50 \%$ of subjects infected with $C$. pneumoniae fail to develop antibodies, and most of these patients are under 5 years of age [29-31]. Interestingly, also two out of the three children with PCR positive for C. pneumoniae DNA and no serologic evidence of acute infection were younger than 5 years of age.

Moreover, 11 children showed mixed acute $M$. pneumoniae and $C$. pneumoniae infection. Mixed infections are common in children with respiratory syndromes but their clinical implications are not clear [32]. In a recent study, HeISKANEN-Kosma et al. [33] showed that mixed chlamydial-mycoplasmal infections constituted $20 \%$ of $M$. pneumoniae infections and as many as $35 \%$ of $C$. pneumoniae infections. The important unanswered question in this regard is whether one pathogen simply facilitates the penetration of the other pathogen, or whether both truly cause pneumonia. Moreover, it is not known if the combination of $M$. pneumoniae and $C$. pneumoniae lead to a more severe clinical illness.

In conclusion, this study shows that, although they are commonly used to do so, neither clinical findings nor laboratory parameters such as white blood cell count, serum C-reactive protein and erythrocyte sedimentation rate were informative for distinction of $M$. pneumoniae and/or $C$. pneumoniae infection in children with pneumonia. Radiological findings also have a limited capacity to differentiate these pathogens.
The priorities for future research include the development of rapid, easily accessible and cost-effective diagnostic tests useful for each episode of pneumonia in children.

Participants in the Mowgli Study Group: R. Longhi, R.M. Grasso (Sant'Anna Hospital, Como); L. Gargantini, P. Cazzaniga (Treviglio Hospital, Treviglio); R. Vaccaro, S. Troiani (Policlinico Monteluce Hospital, Perugia); L. Titone, A. Cascio (Santa Cristina Hospital, Palermo); F. Tancredi, L. Tarallo (S.ma Annunziata Hospital, Napoli); C. Navone, C. Debbia (Santa Corona Hospital, Pietra Ligure); S. Cocuzza, M. Raggi (San Lazzaro Hospital, Alba); L. Zannino (Santo Spirito Hospital, Bra); L. Nespoli, E. Ossola (Del Ponte Hospital, Varese); G. Bona, B. Ronchi (Maggiore della Carità Hospital, Novara); S. Bernasconi, L. Iughetti (Policlinico Hospital, Modena); F. Schettini, N. Rigillo, F. Amendola, L. Mappa (Policlinico Hospital, Bari); S. Volpato, A. Voghenzi (Sant'Anna Hospital, Ferrara); G. Caramia, E. Ruffini (Salesi Hospital, Ancona); I. Barberi, G. Lombardo, S. Gitto, T. Sirchia (Policlinico Hospital, Messina); F. Cordelli, R. Brutti (Belcolle Hospital, Viterbo); S. Catania, C. Ajassa (Policlinico Umberto I Hospital, Roma); S. Santovito (Bosco Hospital, Torino).

\footnotetext{
Acknowledgements. We thank Cristina Arosio, Valentina Popescu Janu, Roberta Droghetti, Giorgio Paizis and Maria Teresa Panza for their substantial contributions to this study.
} 


\section{References}

1. File TM, Tan JS, Plouffe JF. The role of atypical pathogens: Mycoplasma pneumoniae, Chlamydia pneumoniae, and Legionella pneumophila in respiratory infection. Infect Dis Clin North Am 1998; 12: $569-592$.

2. Block S, Hedrick J, Hammerschlag MR, Cassell GH, Craft JC. Mycoplasma pneumoniae and Chlamydia pneumoniae in pediatric community-acquired pneumonia: comparative efficacy and safety of clarithromycin vs. erythromycin ethylsuccinate. Pediatr Infect Dis J 1995; 14: 471 - 477.

3. Hammerschlag MR. Atypical pneumonias in children. Adv Pediatr Infect Dis 1995; 10: 1-39.

4. Cunningham AF, Johnston SL, Julious SA, Lampe FC, Ward ME. Chronic Chlamydia pneumoniae infection and asthma exacerbations in children. Eur Respir J 1998; 11: 345-349.

5. Clyde WA Jr. Clinical overview of typical Mycoplasma pneumoniae infections. Clin Infect Dis 1993; 17 $32-36$.

6. Harris JS, Kolokathis A, Campbell M, Cassell GH, Hammerschlag MR. Safety and efficacy of azithromycin in the treatment of communityacquired pneumonia in children. Pediatr Infect Dis $J$ 1998; 17: 865-871.

7. Principi N, Esposito S. Comparative tolerability of erythromycin and newer macrolide antibacterials in paediatric patients. Drug Safety 1999; 20: 25-41.

8. Dai Y, Foy HM, Zhu Z, Chen B, Tong F. Respiratory rate and signs in roentgenographically confirmed pneumonia among children in China. Pediatr Infect Dis $J$ 1995; 14: 48 - 50 .

9. Tan TQ, Mason Jr EO, Barson WJ, et al. Clinical characteristics and outcome of children with pneumonia attributable to penicillin-susceptible and penicillin-nonsusceptible Streptococcus pneumoniae. Pediatrics 1998; 102: $1369-1375$.

10. Nohynek H, Valkeila E, Leinonen M, Eskola J. Erythrocyte sedimentation rate, white blood cell count and serum C-reactive protein in assessing etiologic diagnosis of acute lower respiratory tract infections in children. Pediatr Infect Dis $J$ 1995; 14 : 484-490.

11. Korppi M, Heiskanen-Kosma T, Leinonen M. White blood cells, C-reactive protein and erythrocyte sedimentation rate in pneumococcal pneumonia in children. Eur Respir J 1997; 10: 1125-1129.

12. Korppi M, Kiekara O, Heiskanen-Kosma T, Soimakallio S. Comparison of radiological findings and microbial aetiology of childhood pneumonia. Acta Paediatr 1993; 82: 360-363.

13. Kiekara O, Korppi M, Tanska S, Soimakallio S. Radiological diagnosis of pneumonia in children. Ann Med 1996; 28: 69-72.

14. Thomson RB. Laboratory diagnosis of respiratory infections. Curr Opin Infect Dis 1999; 12: $115-$ 119.

15. Menéndez $\mathrm{R}$, Còrdoba $\mathrm{J}$, de la Cuadra $\mathrm{P}$, et al. Value of the polymerase chain reaction assay in noninvasive respiratory samples for diagnosis of communityacquired pneumonia. Am J Respir Crit Care Med 1999; 159: $1868-1873$.

16. Abele-Horn $\mathrm{M}$, Busch $\mathrm{U}$, Nitzschiko $\mathrm{H}$, et al. Molecular approaches to diagnosis of pulmonary diseases due to Mycoplasma pneumoniae. J Clin Microbiol 1998; 36: 548-551.

17. Blasi F, Boman J, Esposito G, et al. Chlamydia pneumoniae DNA detection in peripheral blood mononuclear cells is predictive of vascular infection. J Infect Dis 1999; 180: 2074-2076.

18. Davies HD, Wang EEL, Manson D, Babyn P, Shuckett B. Reliability of the chest radiograph in the diagnosis of lower respiratory infections in young children. Pediatr Infect Dis $J$ 1996; 15: 600-604.

19. File TM Jr, Tan JS, Murphy DP. Atypical pneumonia syndrome. Prim Care 1981; 8: 673-694.

20. Woodhead MA, MacFarlane JT. Comparative clinical laboratory features on legionella with pneumococcal and mycoplasma pneumonias. Br J Dis Chest 1987; 81: $133-139$.

21. Heiskanen-Kosma T, Korppi M, Jokinen C, et al. Etiology of childhood pneumonia: serologic results of a prospective, population-based study. Pediatr Infect Dis $J$ 1998; 17: 986-991.

22. Korppi M, Heiskanen-Kosma $\mathrm{T}$, Jaloner E, et al. Aetiology of community-acquired pneumonia in children treated in hospital. Eur J Pediatr 1993; 152: 24-30.

23. Wubbel L, Muniz L, Ahmed A, et al. Etiology and treatment of community-acquired pneumonia in ambulatory children. Pediatr Infect Dis J 1999; 18: $98-104$.

24. Isaacs D. Problems in determining the etiology of community-acquired childhood pneumonia. Pediatr Infect Dis J 1989; 8: 143-148.

25. Korppi M, Kröger L, Laitinen M. White blood cell and differential counts in acute respiratory viral and bacterial infections in children. Scand $J$ Infect Dis 1993; 25: 435-440.

26. Peltola H, Jaakkola M. Serious bacterial infections: Creactive protein as a serial index of severity. Clin Pediatr 1988; 27: $532-537$.

27. Courtoy I, Lande AE, Turner RB. Accuracy of radiographic differentiation of bacterial from nonbacterial pneumonia. Clin Pediatr 1989; 28: 261-264.

28. Friis B, Eiken M, Hornsleth A, Jensen A. Chest X-ray appearances in pneumonia and bronchiolitis. Acta Paediatr Scand 1990; 79: 219-225.

29. Normann E, Gnarpe J, Gnarpe H, Wettergren B. Chlamydia pneumoniae in children with acute respiratory tract infections. Acta Paediatr 1998; 87: $23-27$.

30. Falck G, Gnarpe J, Gnarpe H. Prevalence of Chlamydia pneumoniae in healthy children and in children with respiratory tract infections. Pediatr Infect Dis $J$ 1997; 16: 549-554.

31. Kutlin A, Roblin PM, Hammerschlag MR. Antibody response to Chlamydia pneumoniae infection in children with respiratory illness. J Infect Dis 1998; 177: $720-724$.

32. Korppi M, Leinonen M, Makela PH, Launiala K. Bacterial co-infection in children hospitalized with respiratory syncytial infections. Pediatr Infect Dis $J$ 1989; 8: 687-692.

33. Heiskanen-Kosma T, Korppi M, Laurila A, Jokinen C, Kleemola M, Saikku P. Chlamydia pneumoniae is an important cause of community-acquired pneumonia in school-aged children: serological results of a prospective, population-based study. Scand J Infect Dis 1999; 31: 255-259. 